\title{
Deep-Learning Convolutional Neural Networks Accurately Classify Genetic Mutations in Gliomas
}

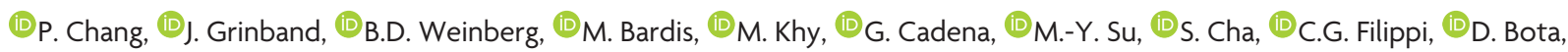

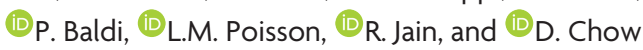

\begin{abstract}
BACKGROUND AND PURPOSE: The World Health Organization has recently placed new emphasis on the integration of genetic information for gliomas. While tissue sampling remains the criterion standard, noninvasive imaging techniques may provide complimentary insight into clinically relevant genetic mutations. Our aim was to train a convolutional neural network to independently predict underlying molecular genetic mutation status in gliomas with high accuracy and identify the most predictive imaging features for each mutation.
\end{abstract}

MATERIALS AND METHODS: MR imaging data and molecular information were retrospectively obtained from The Cancer Imaging Archives for 259 patients with either low- or high-grade gliomas. A convolutional neural network was trained to classify isocitrate dehydrogenase 1 (IDH7) mutation status, 1p/19q codeletion, and O6-methylguanine-DNA methyltransferase (MGMT) promotor methylation status. Principal component analysis of the final convolutional neural network layer was used to extract the key imaging features critical for successful classification.

RESULTS: Classification had high accuracy: IDH1 mutation status, 94\%; 1p/19q codeletion, 92\%; and MGMT promotor methylation status, $83 \%$. Each genetic category was also associated with distinctive imaging features such as definition of tumor margins, T1 and FLAIR suppression, extent of edema, extent of necrosis, and textural features.

CONCLUSIONS: Our results indicate that for The Cancer Imaging Archives dataset, machine-learning approaches allow classification of individual genetic mutations of both low- and high-grade gliomas. We show that relevant MR imaging features acquired from an added dimensionality-reduction technique demonstrate that neural networks are capable of learning key imaging components without prior feature selection or human-directed training.

ABBREVIATIONS: CNN = convolutional neural network; IDH = isocitrate dehydrogenase; $M G M T=$ O6-methylguanine-DNA methyltransferase; VASARI = Visually AcceSAble Rembrandt Images

$D^{i}$ iffuse infiltrating gliomas are a heterogeneous group of primary tumors with highly variable imaging characteristics, response to therapy, clinical course, and prognoses. This well-

Received November 29, 2017; accepted after revision March 20, 2018.

From the Department of Radiology (P.C., S.C.), University of California, San Francisco, San Francisco, California; Department of Radiology (J.G.), Columbia University, New York, New York; Department of Radiology (B.D.W.), Emory University School of Medicine, Atlanta, Georgia; Departments of Radiology (M.B., M.K., M.Y.S., D.C.), Neurosurgery (G.C.), and Neuro-Oncology (D.B.) and School of Information and Computer Sciences (P.B.), University of California, Irvine, Irvine, California; Department of Radiology (C.G.F.), North Shore University Hospital, Long Island, New York; Department of Public Health Sciences (L.M.P.), Henry Ford Health System, Detroit, Michigan; and Departments of Radiology and Neurosurgery (R.J.) New York University, New York, New York.

This work was supported by Canon Medical Systems USA. The work of P.B. was supported, in part, by the following grants: Defense Advanced Research Projects Agency D17AP00002 and National Institutes of Health GM123558. The work of P.C. was in part supported by the National Institutes of Health training grant T32EB001631.

Please address correspondence to Daniel Chow, MD, University of California, Irvine Medical Center, 101 The City Drive South, Douglas Hospital, Route 140, Room 0115, Orange, CA 92868-3201; e-mail: chowd3@uci.edu; @DanChow01 known heterogeneity is, in part, attributed to the multiple variations in the genetic and epigenetic mutations that occur early in tumorigenesis. ${ }^{1}$ For example, isocitrate dehydrogenase 1 and/or 2 (IDH1 and/or 2)-mutant glioblastomas demonstrate significantly improved survivorship compared with $I D H$ wild-type glioblastomas (31 months versus 15 months). ${ }^{2,3}$ Similarly, patients with anaplastic oligodendrogliomas with $1 \mathrm{p} / 19 \mathrm{q}$ codeletion benefit from combined procarbazine/lomustine/vincristine therapy and radiation therapy compared with patients without the mutation. ${ }^{4,5}$ Regarding chemotherapy response, glioblastomas with O6-methylguanine-DNA methyltransferase (MGMT) promoter methylation demonstrate improved response to the combination of temozolomide and radiation therapy versus radiation therapy

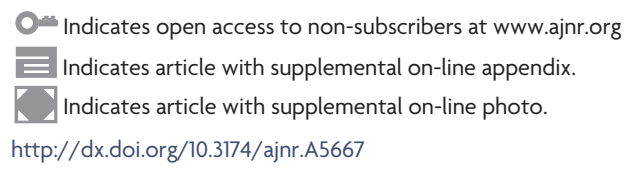


alone (21.7 versus 15.3 months). ${ }^{6}$ The World Health Organization has recently placed new emphasis on the integration of genetic and molecular information for CNS tumor-classification schemes, including IDH1 status and $1 \mathrm{p} / 19 \mathrm{q}$ codeletion and several other molecular or genetic markers. ${ }^{7}$ Thus, knowledge of tumoral genetic information is needed to accurately monitor patients with gliomas and guide personalized therapies.

At present, information regarding underlying genetic and molecular alterations of gliomas is based on analysis of tumor tissue obtained during an operation. However, although high-grade gliomas are known to infiltrate widely into the surrounding nonenhancing peritumoral region, ${ }^{8}$ biopsies are often limited to the easily accessible areas of the enhancing tumor. Additionally, molecular genetic testing can be costly or not widely available, and the results may take weeks, thereby delaying important therapeutic decisions. Noninvasive imaging techniques that can provide complimentary insight into clinically relevant genetic mutations may expedite and coordinate care among clinicians, minimizing these delays.

MR imaging can noninvasively assess the entire tumor, allowing both a global and regional (voxelwise) characterization of molecular genetics, in contrast to the spatially limited assessment of tissue biopsy. Specifically, both spatial and temporal variations in genetic expression are known to result in heterogeneous alterations in tumor biology, including changes in angiogenesis, cellular proliferation, cellular invasion, and apoptosis. ${ }^{9}$ These biologic changes are reflected in the complex imaging features of gliomas, manifest by varying degrees of enhancement, infiltration, hemorrhage, reduced diffusion, edema, and necrosis. Attempts to standardize visual interpretation of malignant gliomas for tissue classification have led to the Visually AcceSAble Rembrandt Images (VASARI) feature set, a rule-based lexicon to improve the reproducibility of interpretation. ${ }^{10}$ However, a limitation of such approaches is the need for a priori feature selection and human visual interpretation, which innately distills a complex dataset of over a million voxels per MR imaging to a handful of numeric descriptors-a "big data" challenge.

The purpose of this study was to classify genetic variations of diffuse infiltrating gliomas using deep-learning/machine-learning approaches implemented with convolutional neural networks (CNNs). CNN approaches model the animal visual cortex by applying a feed-forward artificial neural network to simulate multiple layers of neurons organized in overlapping regions within a visual field, with each layer acting to transform the raw input image into more complex, hierarchic, and abstract representations. ${ }^{11}$ Thus, it is natural to consider applying deep-learning methods to biomedical images. We hypothesized the following: 1) A CNN can be trained to independently predict underlying molecular genetic mutation status in gliomas with high accuracy, and 2) a trained $\mathrm{CNN}$ can identify predictive imaging features for a given mutation.

\section{MATERIALS AND METHODS Subjects}

MR imaging data were retrospectively obtained from The Cancer Imaging Archives for patients with either low- or high-grade gliomas. ${ }^{12}$ Corresponding molecular genetic information was ob- tained from The Cancer Genome Atlas. Only patients with full preoperative MR imaging, including T2, FLAIR, and T1-weighted pre- and postcontrast acquisitions, were included in the analysis. Corresponding molecular information for each patient was obtained, including $I D H 1$ status, $1 \mathrm{p} / 19 \mathrm{q}$ codeletion, and MGMT promoter methylation. ${ }^{13}$

\section{Image Preprocessing}

For each patient, all imaging modalities were coregistered using the FMRIB Linear Image Registration Tool (FLIRT; http://www. fmrib.ox.ac.uk/fsl/fslwiki/FLIRT). ${ }^{14,15}$ Registration was implemented with a linear affine transformation algorithm using $12 d f$, trilinear interpolation, and a mutual-information cost function. The reference volume for coregistration was the highest resolution sequence, most commonly the postcontrast T1-weighted acquisition. The average time for coregistration was approximately 1 minute per volume. On a typical multicentral processing unit core workstation, the required total of 3 registrations per patient can be performed simultaneously as separate processes, thus allowing all modalities to be coregistered in approximately 1 minute.

Each input technique was independently normalized using $z$ score values ( $\mu=0, \sigma=1)$. From these, a custom in-house fully automated whole-brain extraction tool-based $3 \mathrm{D}$ convolutional neural network was used to remove extracranial structures. Next, a fully automated brain tumor segmentation tool was used to identify lesion margins. This algorithm was the top-performing tool as evaluated in the international 2016 Multimodal Brain Tumor Segmentation Challenge. ${ }^{16}$ It is based on a serial fully convolutional neural network architecture with residual connections and performs whole-tumor segmentation in approximately $1 \mathrm{sec}-$ ond. These masks were used to generate cropped slice-by-slice images of the tumor on all modalities, each of which were subsequently resized to a $32 \times 32 \times 4$ input.

No other form of preprocessing was necessary for this study. Specifically, the flexibility of CNNs allows robust classification, even in the absence of conventional image-preprocessing steps such as histogram normalization or bias field correction.

\section{Convolutional Neural Networks}

$\mathrm{CNNs}$ are an adaption of the traditional artificial neural network architecture whereby banks of 2D convolutional filter parameters and nonlinear activation functions act as a mapping function to transform a multidimensional input image into a desired output. ${ }^{17}$ Network overview and details are provided in the On-line Appendix.

\section{Feature Analysis}

The final feature vector produced by a neural network through serial convolutions often encodes for redundant information, given the flexibility of the algorithm to choose any feature necessary to produce accurate classification. In the architecture used for this study, this means that many of 64 features in the final hidden layer will be highly correlated to each other. To decompose this encoded information and gain insight into features learned by the algorithm, we applied principal component analysis to the final feature vector with various dimensionally reduced 
A
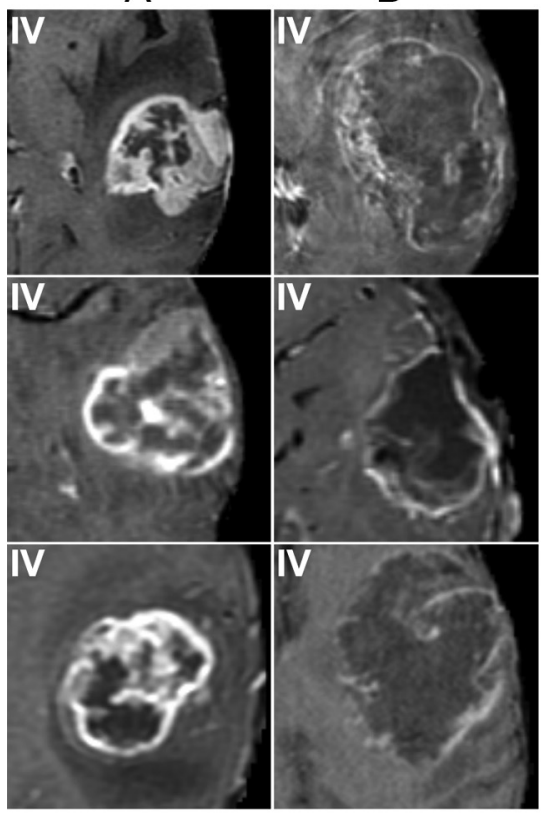

IDH1-Wild Types

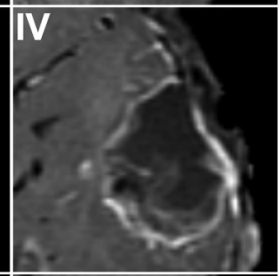

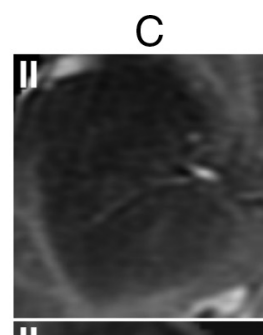
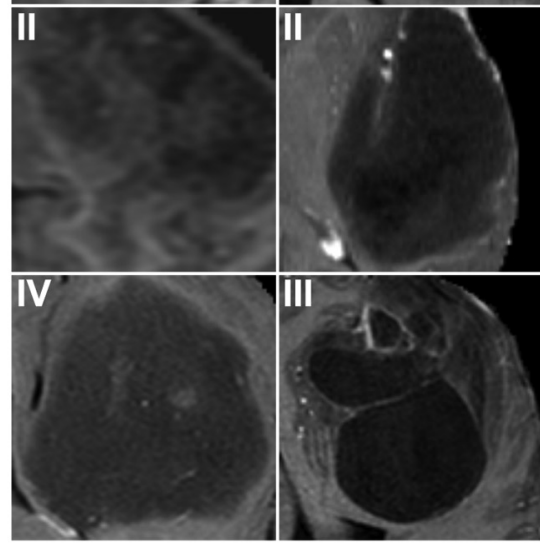

IDH1-Mutant Types

FIG 1. T1 postcontrast features most highly correlated with IDH7 mutation status. Prototypical cases as identified by our convolutional neural network imaging features associated with IDH wild types $(A$ and $C)$ and mutation $(B$ and $D)$. Specifically, IDH wild types demonstrate thick and irregular enhancement $(A)$ or thin, irregular poorly marinated peripheral enhancement $(B)$. In contrast, patients with IDH mutation demonstrate absent or minimal enhancement $(C)$ with well-defined tumor margins $(D)$.

A

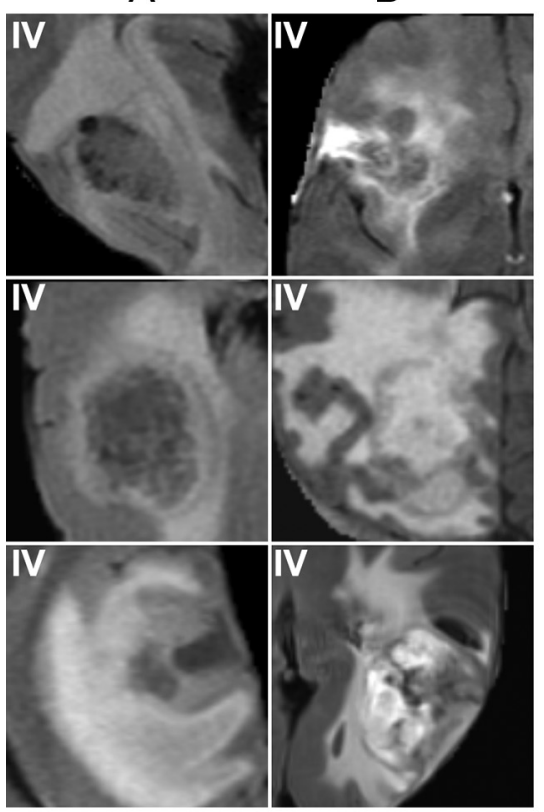

IDH1-Wild Types

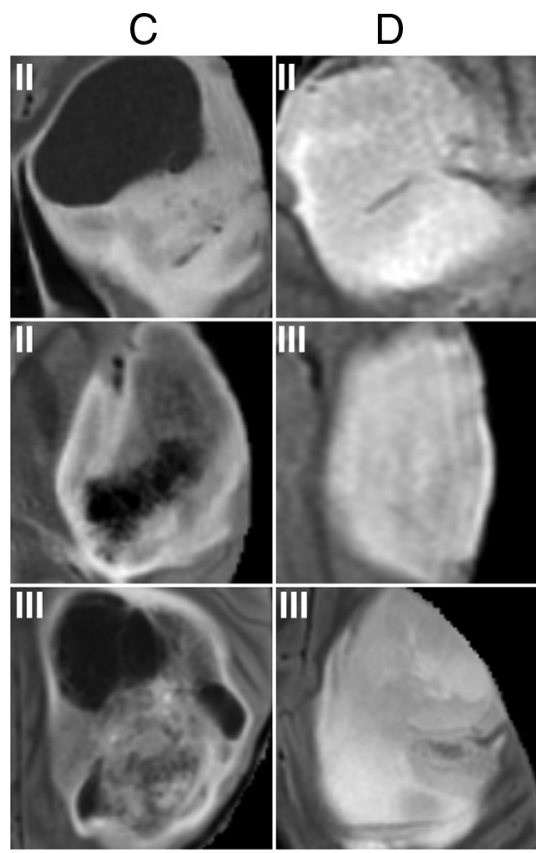

IDH1-Mutant Types

FIG 2. FLAIR features most highly correlated with $I D H 1$ mutation status. Prototypical cases as identified by our convolutional neural network imaging features are associated with IDH wild types $(A$ and $B)$ and mutation $(C$ and $D)$. IDH wild types demonstrate infiltrative patterns of edema, seen as more irregular $(A)$ and ill-defined $(D)$ margins of FLAIR signal abnormality. Patients with IDH mutation demonstrate central areas of cysts with FLAIR suppression $(C)$ and well-defined tumor margins (D).

subspaces, $L \epsilon .^{1,5}$ By means of this approach, the principal component analysis-reduced features, whose weights have the largest absolute value magnitude with respect to the final classification, can be identified. These features can be interpreted as those automatically learned by the algorithm that are most influential in classification of any given mutation status. These final imaging features identified by the algorithm are shown in Figs 1-4.

\section{Statistical Analysis}

To evaluate overall per-patient accuracy, we pooled mean softmax scores across all image slices, with a threshold of 0.5 used to determine mutation classification. As an example of $I D H$ status, an average softmax score of $>0.5$ represents prediction of mutant status, while an average softmax score of $<0.5$ represents prediction of wild-type status. While a softmax score of 0.5 is the standard threshold for neural network classification, it is possible to arbitrarily change this cutoff to between 0 and 1 to alter the sensitivity and specificity of the network. By means of this approach, overall algorithm performance on a wide range of thresholds is reported as an area under the curve calculation for each mutation.

To assess algorithm generalization, we used a 5-fold cross-validation approach. For each of the 5 experimental iterations, $20 \%$ of the data were used for validation, while the remaining $80 \%$ of the data were used for training. Results are reported for only patients in the validation cohort after 5-fold cross-validation.

\section{RESULTS \\ Subjects}

A total of 5259 axial slices of tumor from 259 patients with gliomas (135 men/122 women/2 unknown; mean age, 53.2 years; mean survival, 18.8 years) were included for analysis. The distribution of gliomas included the following: $21.2 \%$ (55/259) grade II, 22.8\% (59/259) grade III, and 55.2\% (143/259) grade IV gliomas. There were 2 gliomas that had available genetic information but no World Health Organization grade assignment. IDH1 mutant and wild-type tumors accounted for $45.9 \%$ (119/259) and $54.1 \%(140 / 259)$ of patients, respectively; $1 \mathrm{p} / 19 \mathrm{q}$ codeletions and nondele- 
A

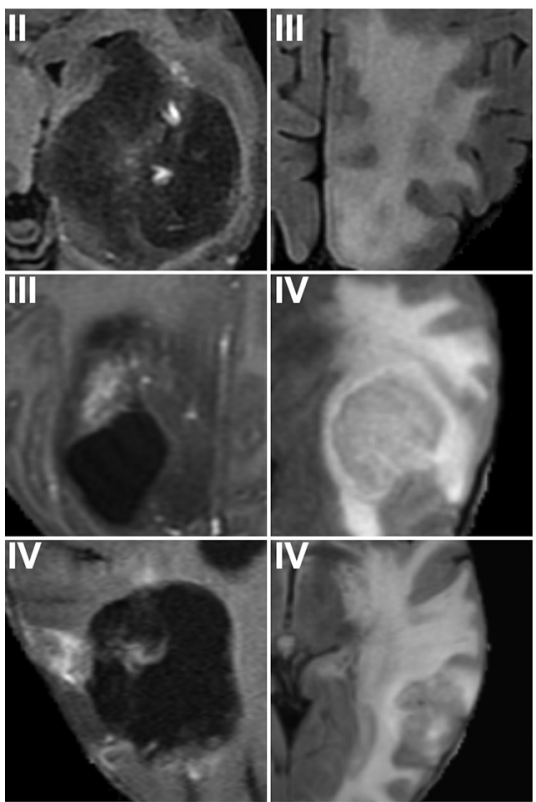

1p/19q (-) co deletion

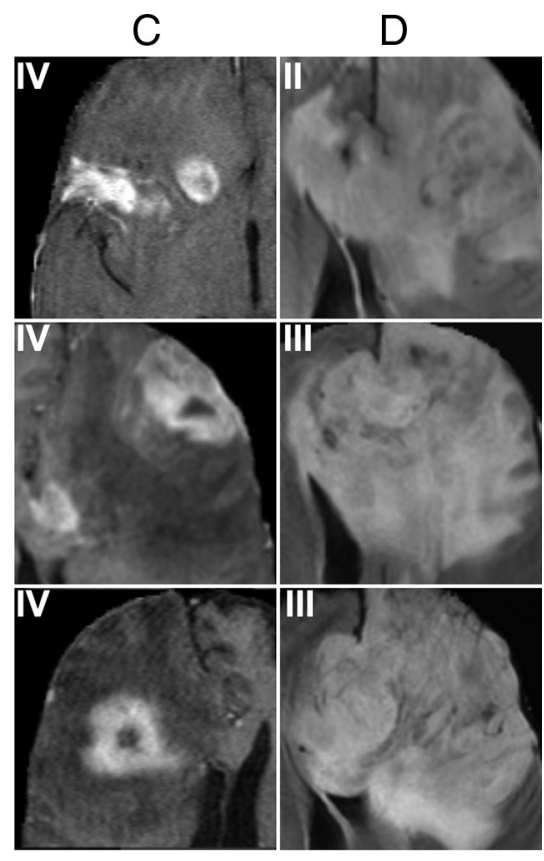

1p/19q (+) co deletion
FIG 3. MR imaging features most highly correlated with $1 p / 19 q$ codeletion. Prototypical cases as identified by our convolutional neural network imaging features are associated with $1 p / 19 q$ absence $(A$ and $B)$ and presence $(C$ and $D)$ codeletion status. Features predictive of the absence of $1 \mathrm{p} / 19 \mathrm{q}$ codeletion are poor enhancement $(A)$ and increased vasogenic edema $(B)$. Features predictive of the presence of $1 p / 19 q$ codeletion are increased enhancement $(C)$ and a left frontal predominance and ill-defined FLAIR margins with mass effect $(D)$.
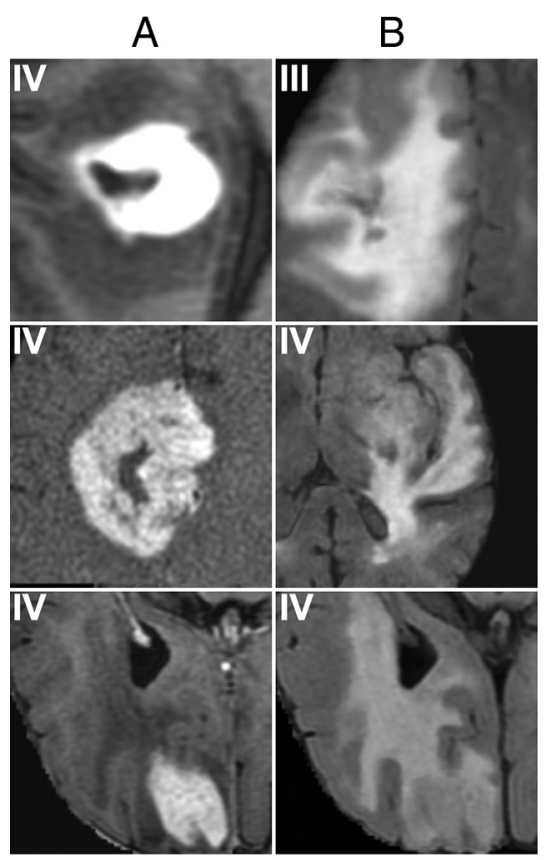

MGMT unmethylated
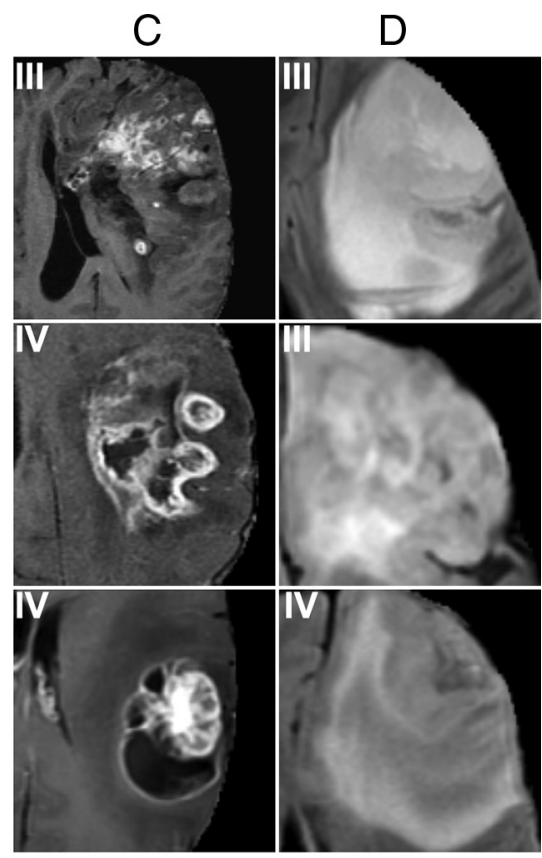

\section{MGMT methylated}

FIG 4. Features most highly correlated with MGMT methylation. Prototypical cases as identified by our convolutional neural network imaging features associated with unmethylated $(A$ and $B)$ and methylated $(C$ and $D)$ MGMT statuses. Features predictive of MGMT unmethylated status include thick enhancement with central necrosis $(A)$ with infiltrative edema patterns $(B)$. In contrast, features predictive of MGMT promoter methylated status include nodular and heterogeneous enhancement $(C)$ with masslike FLAIR edema $(D)$. tions accounted for $12.0 \%(31 / 259)$ and $88.0 \%(228 / 259)$ of patients, respectively. MGMT promoter methylated and unmethylated accounted for $56.4 \%$ $(146 / 259)$ and $43.6 \%(113 / 259)$ of patients, respectively. The mean tumor size determined by automated segmentation masks was $105.6 \mathrm{~cm}^{3}$.

\section{CNN Accuracy}

Overall, the algorithm correctly predicted IDH1 mutation (mean, 94\%; range between cross validations, 90\%96\%), 1p/19q codeletion (mean, 92\%; range, $88 \%-95 \%$ ), and MGMT promoter methylation (mean, 83\%; range, 76\%$88 \%$ ) on 5 -fold cross-validation. The area under the curve for $I D H$ mutation (mean, 0.91 ; range, $0.89-0.92), 1 \mathrm{p} / 19 \mathrm{q}$ codeletion (mean, 0.88; range, 0.85-0.90), and MGMT promoter methylation (mean, 0.81 ; range, $0.76-0.84$ ) also reflected high performance. The CNN was trained for 25,000 iterations (approximately $3000 \mathrm{ep}$ ochs with batch sizes ranging from 12 to 48) before convergence. A single forward pass during test time for classification of new cases can be achieved in 0.0043 seconds. Overall, the imaging workflow takes 5.12 seconds per patient $(4-5$ seconds for detection and preprocessing and the remaining 0.0043 seconds for classification).

\section{Feature Analysis}

For IDH1 mutation (Figs 1 and 2), the most predictive features were the following: absent or minimal areas of enhancement (the presence of a larger portion of nonenhancing tumor), central areas of cysts with low T1 and FLAIR suppression, and well-defined tumor margins. By comparison, IDH1 wildtype tumors tended to demonstrate a larger portion of enhancing tumor with thick enhancement; thin, irregular, poorly marginated peripheral enhancement with central necrosis; and an infiltrative pattern of edema (seen as more irregular and ill-defined margins of T2/ FLAIR signal abnormality).

For 1p19 codeletion (Fig 3), the most predictive features were left frontal lobe location, ill-defined tumor margins, and larger portion of enhancement. Compared with either IDH1 mutation or MGMT promoter methylation, many features learned by the CNN for 1p19 
codeletion were highly correlated to each other; this finding resulted in an overall smaller number of differentiable features.

For MGMT promoter methylation (Fig 4), the most predictive features were a heterogeneous, nodular enhancement; the presence of an eccentric cyst; more masslike edema (larger lesions with a higher portion of nonenhancing tumor component) with cortical involvement; and slight frontal and superficial temporal predominance. By comparison, unmethylated tumors tended to demonstrate thin rim enhancement, central areas of necrosis, solid enhancement, more vasogenic edema, and a slight, deep temporal predominance.

\section{DISCUSSION}

The variability of clinical outcomes in patients with diffuse infiltrating gliomas is, in part, predicated on molecular and genetic heterogeneity, which has spurred the development and study of noninvasive tools to better classify these tumors. In this study, we used a deep-learning approach to classify individual somatic mutations of diffuse infiltrating gliomas, World Health Organization II-IV, including IDH1 status; 1p/19q codeletion; and the presence of MGMT promoter methylation with an accuracy of $94 \%(90 \%-$ 96\%), 92\% (88\%-95\%), and 83\% (76\%-88\%), respectively. We were able to implement the entire preprocessing pipeline from tumor detection to tissue segmentation to mutation classification without human supervision. Furthermore, neural networks have been criticized for being "black boxes" that generate uninterpretable feature vectors, which limits insight into the underlying mechanism for image classification. In this study, we applied a dimensionality-reduction approach to visually display the highest ranking features of each mutation category.

Molecular analysis of tumors has significantly impacted the diagnosis of glial tumors, with important implications for both prognosis and therapy guidance. The recently published 2016 World Health Organization Classification of Tumors of the Central Nervous System included several molecular genetic alterations as important features of tumor classification. ${ }^{7}$ One of the significant changes has been in the classification of oligodendrogliomas, in which mutations of IDH1 or 2 and 1p/19q codeletion are the defining and diagnostic markers. Additionally, hypermethylation of the MGMT promoter, an enzyme involved in DNA de-alkylation and mediation of DNA damage, is a positive prognostic factor. ${ }^{6}$ Patients with a methylated MGMT promoter have improved survival and better response to radiation therapy with concurrent temozolomide. ${ }^{6,18}$

Prior classic machine-learning approaches for linking imaging features to these genetic alternations in gliomas have typically relied on human-derived feature extraction such as textural analysis approaches or rule-based systems such as VASARI. For example, Ryu et $\mathrm{al}^{19}$ applied texture analysis to evaluate glioma heterogeneity to distinguish low- and high-grade gliomas with an $80 \%$ accuracy. Additionally, Drabycz et $\mathrm{al}^{20}$ described a textural analysis approach to classifying MGMT promoter methylation status in patients with glioblastomas with $71 \%$ accuracy. More recently, Kanas et $\mathrm{al}^{21}$ achieved a $74 \%$ accuracy in distinguishing the MGMT promoter methylation status from gliomas acquired from The Cancer Genome Atlas using a multivariate prediction model based on qualitative imaging features from the VASARI lexicon.
While these approaches have improved the reproducibility and accuracy of classification, the need for manual a priori feature selection remains an inherently limiting factor, a process dependent on expert opinion and an assumption of relevant features. ${ }^{22}$

As a result, there has been a recent paradigm shift toward endto-end machine learning using $\mathrm{CNNs}$, which are rapidly outperforming conventional benchmarks on various computer vision tasks. ${ }^{11,23}$ These models are capable of automatically identifying patterns in complex imaging datasets, thus combining both feature selection and classification into 1 algorithm and removing the need for direct human interaction during the training process. With deep-learning approaches, classification error rates of popular computer vision benchmarks have been significantly lower and now outperform humans on the same task. ${ }^{24-26}$

Recent use of CNNs has started to yield promising results in multiple medical imaging disciplines, including the detection of pulmonary nodules, ${ }^{27}$ colon cancer, ${ }^{28}$ and cerebral microbleeds. ${ }^{29}$ For example, Lakhani and Sundaram ${ }^{30}$ applied a CNN approach to automatically identify patients with pulmonary tuberculosis with an area under the curve of 0.99 , allowing radiologists to achieve a $97 \%$ sensitivity and $100 \%$ specificity. This outcome is in comparison with an area under the curve of up to 0.84 using classic machine-learning approaches such as texture and shape analysis. ${ }^{31}$ Additionally, Chang et $\mathrm{al}^{32}$ developed a CNN approach to automatically identify and count tumor cells from localized biopsy samples of patients with glioblastomas with an accuracy of $96.2 \%$. Zhang et $\mathrm{al}^{33}$ observed that a CNN approach performed significantly better than other techniques for brain segmentation in infants, including random forest, support vector machine, coupled level sets, and most voting.

Given the potential advantages of deep learning, a few studies have also started to explore the use of CNN-based approaches in the determination of glioma mutation status from MR imaging. Recently, Chang et $\mathrm{al}^{34}$ used a 34-layer residual neural network to predict $I D H$ status with up to $89 \%$ accuracy using MR imaging in combination with patient age. Compared with the current study, the network used by Chang et al has several million parameters ( $>1$ order magnitude larger than the customized network used in this study), in part limiting overall accuracy through compensatory measures needed to prevent overfitting. Furthermore, only several prototypical slices of the tumor were used (compared with the entire volume in this study), which were then combined in all 3 orthogonal planes (requiring high-resolution isotropic imaging). Korfiatis et $\mathrm{al}^{35}$ also recently described a 50-layer residual network architecture to predict MGMT status. However, the reported classification accuracy of $94.9 \%$ comprising 2027 of 2612 images (78\%) used for testing contained no tumor at all. Furthermore, the 155 patients used in that study were derived completely from just a single academic center. Finally, in comparison with these prior works, the current study is the first to demonstrate the feasibility of a single neural network architecture to simultaneously predict the status of multiple different mutations (IDH1 status, $1 \mathrm{p} / 19 \mathrm{q}$ codeletion, MGMT promoter methylation) with minimal preprocessing in an efficient, fully automated approach.

Despite high accuracy, a commonly cited limitation of CNNs is the apparent difficulty in understanding the underlying black box analytic engine of a network. Several recent studies, however, 
have proposed novel techniques such as deconvolutional neural networks and occlusion saliency maps to develop a deeper mechanistic understanding of the classification process. ${ }^{36}$ In this study, we introduced a new technique to visualize the imaging features most relevant to the classification of genetic mutation status using principal component analysis as a means of dimensionality reduction and disentanglement of the final feature vector layer. This approach is useful in medical imaging domains in which the differentiating characteristics of the various disease classes may not be well-established, helping to identify clusters of imaging findings that can be used to guide practicing physicians (Figs 1-4).

In general, the clusters of imaging features identified by the neural network in this study represent a composite of various qualitative descriptions found elsewhere in the literature. For example, MR imaging features predictive of IDH1 mutant status included absent or minimal areas of enhancement, central areas of cystlike necrosis with low T1 and FLAIR suppression, and welldefined tumor margins. This result is in line with existing literature, in which IDH1 mutants have been reported to demonstrate absent or minimal enhancement ${ }^{37-39}$ and well-defined tumor margins. ${ }^{38,40}$ By contrast, we observed that IDH1 wild-type tumors demonstrated thick and irregular enhancement with an infiltrative pattern of edema.

For $1 \mathrm{p} / 19 \mathrm{q}$ codeletion, the most predictive features were frontal lobe location, ill-defined tumor margins, and increased enhancement. This finding is also in line with existing literature, which has demonstrated that tumors with $1 \mathrm{p} / 19 \mathrm{q}$ codeletion are more likely to be found in the frontal cortex. ${ }^{41}$ Additionally, Sonoda et $\mathrm{al}^{39}$ demonstrated that codeleted tumors are more likely to show contrast enhancement. Finally, the margins of $1 \mathrm{p} /$ $19 \mathrm{q}$ codeleted tumors have also been characterized as poorly circumscribed. ${ }^{42}$

With regard to MGMT promoter methylation, the most predictive features were a mixed, nodular enhancement; the presence of an eccentric cyst or area of necrosis; more masslike edema with cortical involvement; and slight frontal and superficial temporal predominance. Existing literature has similarly observed that tumors with MGMT promoter methylation tend to have a frontal lobe location $^{43,44}$ (often colocalization with the IDH1 mutation in this region ${ }^{43}$ ) and the presence of an eccentric necrotic cyst. ${ }^{20,45}$ By comparison, we observed that nonmethylated tumors tended to demonstrate rim enhancement with central areas of necrosis. This observation is also congruent with other literature that has used subjective visual assessment, in which nonmethylated tumors are observed to demonstrate either ring enhancement with central necrosis, ${ }^{20,46}$ solid enhancement, ${ }^{21}$ and ill-defined margins. ${ }^{45}$

When one interprets the results of our study, several limitations should be kept in mind. First, this is a relatively small sample size $(n=259)$ compared with the neural network studies within the nonmedical domains, which typically include tens of thousands. To address this limitation, we designed a tailormade neural network architecture with a relatively small number of parameters/layers and high normalization. Additionally, all imaging input was resampled to a relatively small size $(32 \times 32 \times 4)$ to prevent overfitting. Therefore, input for prediction is limited to 4096 voxels on any given slice of tumor as opposed to the potential tens of thousands of voxels. Second, this study is a retrospective study of The Cancer Imaging Archives dataset, a heterogeneous dataset from multiple different contributing sites. However, the success of our network on this dataset suggests that the underlying $\mathrm{CNN}$ approach in this study is capable of handling nonuniform imaging protocols. Last, this study is limited by lack of an independent dataset. While the cross-fold validation technique used in this study ensures that the model generalizes well to held-out cohorts from The Cancer Imaging Archives dataset, generalization to unseen datasets remains to be determined. Future studies will need to expand the training set to include a variety of cancer sites and MR imaging scanners.

\section{CONCLUSIONS}

The results of our study show the feasibility of a deep-learning $\mathrm{CNN}$ approach for the accurate classification of individual genetic mutations of both low- and high-grade gliomas. Furthermore, we demonstrate that the relevant MR imaging features acquired from an added dimensionality-reduction technique are concordant with existing literature, showing that neural networks are capable of learning key imaging components without prior feature selection or human directed training.

Disclosures: Peter Chang—RELATED: Grant: National Institutes of Health (National Institute of Biomedical Imaging and Bioengineering) T32 Training Grant, T32EB001631*. Christopher G. Filippi-UNRELATED: Consultancy: KOL Philips Healthcare, Comments: part of a Key Opinion Leaders consortium in which I have advocated for CNNs in advanced imaging including neoplasms; Payment for Lectures Including Service on Speakers Bureaus: Visiting Professor, Comments: In a talk on advanced imaging of tumor, some of the preliminary work was included. Pierre Baldi-RELATED: Grant: National Institutes of Health*; UNRELATED: Royalties: MIT Press, Cambridge University Press, Wiley. Daniel Chow—RELATED: Grant: funding support from Canon Medical Systems USA. *Money paid to the institution.

\section{REFERENCES}

1. Cohen AL, Holmen SL, Colman H. IDH1 and IDH2 mutations in gliomas. Curr Neurol Neurosci Rep 2013;13:345 CrossRef Medline

2. Nobusawa S, Watanabe T, Kleihues P, et al. IDH1 mutations as molecular signature and predictive factor of secondary glioblastomas. Clin Cancer Res 2009;15:6002-07 CrossRef Medline

3. Yan H, Parsons DW, Jin G, et al. IDH1 and IDH2 mutations in gliomas. N Engl J Med 2009;360:765-73 CrossRef Medline

4. van den Bent MJ, Brandes AA, Taphoorn MJ, et al. Adjuvant procarbazine, lomustine, and vincristine chemotherapy in newly diagnosed anaplastic oligodendroglioma: long-term follow-up of EORTC brain tumor group study 26951. J Clin Oncol 2013;31: 344-50 CrossRef Medline

5. Cairncross G, Wang M, Shaw E, et al. Phase III trial of chemoradiotherapy for anaplastic oligodendroglioma: long-term results of RTOG 9402. J Clin Oncol 2013;31:337-43 CrossRef Medline

6. Hegi ME, Diserens AC, Gorlia T, et al. MGMT gene silencing and benefit from temozolomide in glioblastoma. N Engl J Med 2005;352: 997-1003 CrossRef Medline

7. Louis DN, Perry A, Reifenberger G, et al. The 2016 World Health Organization Classification of Tumors of the Central Nervous System: a summary. Acta Neuropathol 2016;131:803-20 CrossRef Medline

8. Gill BJ, Pisapia DJ, Malone HR, et al. MRI-localized biopsies reveal subtype-specific differences in molecular and cellular composition at the margins of glioblastoma. Proc Natl Acad Sci U S A 2014;111: 12550-55 CrossRef Medline

9. Belden CJ, Valdes PA, Ran C, et al. Genetics of glioblastoma: a window into its imaging and histopathologic variability. Radiographics 2011;31:1717-40 CrossRef Medline 
10. National Cancer Institute. Wiki for the VASARI feature set. https:// wiki.nci.nih.gov/display/CIP/VASARI. Accessed August 30, 2017

11. LeCun Y, Bengio Y, Hinton G. Deep learning. Nature 2015;521: 436-44 CrossRef Medline

12. Clark K, Vendt B, Smith K, et al. The Cancer Imaging Archive (TCIA): maintaining and operating a public information repository. J Digit Imaging 2013;26:1045-57 CrossRef Medline

13. Ceccarelli M, Barthel FP, Malta TM, et al. Molecular profiling reveals biologically discrete subsets and pathways of progression in diffuse glioma. Cell 2016;164:550-63 CrossRef Medline

14. Jenkinson M, Smith S. A global optimisation method for robust affine registration of brain images. Med Image Anal 2001;5:143-56 CrossRef Medline

15. Jenkinson M, Bannister $\mathrm{P}, \mathrm{Brady} \mathrm{M}$, et al. Improved optimization for the robust and accurate linear registration and motion correction of brain images. Neuroimage 2002;17:825-41 CrossRef Medline

16. Chang PD. Fully convolutional deep residual neural networks for brain tumor segmentation. In: Crimi A, Menze B, Maier O, et al, eds. Brainlesion: Glioma, Multiple Sclerosis, Stroke and Traumatic Brain Injuries: Second International Workshop, BrainLes 2016, with the Challenges on BRATS, ISLES and mTOP 2016, Held in Conjunction with MICCAI 2016, Athens, Greece, October 17, 2016, Revised Selected Papers. Cham: Springer-Verlag International Publishing; 2016:108-18

17. LeCun $Y$, Bengio $Y$. Convolutional networks for images, speech, and time-series. In: Arbib MA, ed. The Handbook of Brain Theory and Neural Networks. Cambridge: MIT Press; 1998:255-58

18. Gorlia T, van den Bent MJ, Hegi ME, et al. Nomograms for predicting survival of patients with newly diagnosed glioblastoma: prognostic factor analysis of EORTC and NCIC trial 26981-22981/CE.3. Lancet Oncol 2008;9:29-38 CrossRef Medline

19. Ryu YJ, Choi SH, Park SJ, et al. Glioma: application of whole-tumor texture analysis of diffusion-weighted imaging for the evaluation of tumor heterogeneity. PLoS One 2014;9:e108335 CrossRef Medline

20. Drabycz $S$, Roldán $G$, de Robles $P$, et al. An analysis of image texture, tumor location, and MGMT promoter methylation in glioblastoma using magnetic resonance imaging. Neuroimage 2010;49:1398-405 CrossRef Medline

21. Kanas VG, Zacharaki EI, Thomas GA, et al. Learning MRI-based classification models for MGMT methylation status prediction in glioblastoma. Comput Methods Programs Biomed 2017;140:249-57 CrossRef Medline

22. Kassner A, Thornhill RE. Texture analysis: a review of neurologic MR imaging applications. AJNR Am J Neuroradiol 2010;31:809-16 CrossRef Medline

23. Simonyan K, Vedaldi A, Zisserman A. Deep inside convolutional networks: visualising image classification models and saliency maps. CoRR 2013;abs/1312.6034. https://arxiv.org/abs/1312.6034. Accessed August 30, 2017

24. He K, Zhang X, Ren S, et al. Delving deep into rectifiers: surpassing human-level performance on ImageNet classification. In: Proceedings of the IEEE International Conference on Computer Vision, Santiago, Chili. December 7-13, 2015:1026-34

25. He K, Zhang X, Ren S, et al. Deep residual learning for image recognition. CoRR 2015;abs/1512.03385. https://arxiv.org/abs/1512.03385. Accessed August 30, 2017

26. Krizhevsky A, Sutskever I, Hinton G. ImageNet classification with deep convolutional neural networks. https://www.nvidia.cn/content/tesla/pdf/machine-learning/imagenet-classification-withdeep-convolutional-nn.pdf. Accessed August 30, 2017

27. Setio AA, Ciompi F, Litjens G, et al. Pulmonary nodule detection in CT images: false positive reduction using multi-view convolutional networks. IEEE Trans Med Imaging 2016;35:1160-69 CrossRef Medline

28. Roth $\mathrm{HR}, \mathrm{Lu} \mathrm{L}$, Liu J, et al. Improving computer-aided detection using convolutional neural networks and random view aggregation. IEEE Trans Med Imaging 2016;35:1170-81 CrossRef Medline
29. He K, Zhang X, Ren S, et al. Deep residual learning for image recognition. In: Proceedings of the IEEE Conference on Computer Vision and Pattern Recognition, Las Vegas, Nevada. June 27-30, 2016

30. Lakhani P, Sundaram B. Deep learning at chest radiography: automated classification of pulmonary tuberculosis by using convolutional neural networks. Radiology 2017;284:574-82 CrossRef Medline

31. Pande T, Cohen C, Pai M, et al. Computer-aided detection of pulmonary tuberculosis on digital chest radiographs: a systematic review. Int J Tuberc Lung Dis 2016;20:1226-30 CrossRef Medline

32. Chang PD, Malone HR, Bowden SG, et al. A multiparametric model for mapping cellularity in glioblastoma using radiographically localized biopsies. AJNR Am J Neuroradiol 2017;38:890-98 CrossRef Medline

33. Zhang W, Li R, Deng H, et al. Deep convolutional neural networks for multi-modality isointense infant brain image segmentation. Neuroimage 2015;108:214-24 CrossRef Medline

34. Chang K, Bai HX, Zhou H, et al. Residual convolutional neural network for the determination of IDH status in low- and high-grade gliomas from MR imaging. Clin Cancer Res 2018;24:1073-81 CrossRef Medline

35. Korfiatis P, Kline TL, Lachance DH, et al. Residual deep convolutional neural network predicts MGMT methylation status. J Digit Imaging 2017;30:622-28 CrossRef Medline

36. Zeiler MD, Fergus R. Visualizing and understanding convolutional networks. In: Fleet D, Pajdla T, Schiele B, et al, eds. Proceedings of the European Conference on Computer Vision, Zurich, Switzerland. September 6-12, 2014

37. Carrillo JA, Lai A, Nghiemphu PL, et al. Relationship between tumor enhancement, edema, IDH1 mutational status, MGMT promoter methylation, and survival in glioblastoma. AJNR Am J Neuroradiol 2012;33:1349-55 CrossRef Medline

38. Qi S, Yu L, Li H, et al. Isocitrate dehydrogenase mutation is associated with tumor location and magnetic resonance imaging characteristics in astrocytic neoplasms. Oncol Lett 2014;7:1895-902 CrossRef Medline

39. Sonoda Y, Shibahara I, Kawaguchi T, et al. Association between molecular alterations and tumor location and MRI characteristics in anaplastic gliomas. Brain Tumor Pathol 2015;32:99-104 CrossRef Medline

40. Metellus P, Coulibaly B, Colin C, et al. Absence of IDH mutation identifies a novel radiologic and molecular subtype of WHO grade II gliomas with dismal prognosis. Acta Neuropathol 2010;120: 719-29 CrossRef Medline

41. Xiong J, Tan W, Wen J, et al. Combination of diffusion tensor imaging and conventional MRI correlates with isocitrate dehydrogenase $1 / 2$ mutations but not $1 \mathrm{p} / 19 \mathrm{q}$ genotyping in oligodendroglial tumours. Eur Radiol 2016;26:1705-15 CrossRef Medline

42. Johnson DR, Diehn FE, Giannini C, et al. Genetically defined oligodendroglioma is characterized by indistinct tumor borders at MRI. AJNR Am J Neuroradiol 2017;38:678-84 CrossRef Medline

43. Ellingson BM, Lai A, Harris RJ, et al. Probabilistic radiographic atlas of glioblastoma phenotypes. AJNR Am J Neuroradiol 2013;34: 533-40 CrossRef Medline

44. Paldor I, Pearce FC, Drummond KJ, et al. Frontal glioblastoma multiforme may be biologically distinct from non-frontal and multilobar tumors. J Clin Neurosci 2016;34:128-32 CrossRef Medline

45. Moon WJ, Choi JW, Roh HG, et al. Imaging parameters of high grade gliomas in relation to the MGMT promoter methylation status: the CT, diffusion tensor imaging, and perfusion MR imaging. Neuroradiology 2012;54:555-63 CrossRef Medline

46. Eoli M, Menghi F, Bruzzone MG, et al. Methylation of O6-methylguanine DNA methyltransferase and loss of heterozygosity on $19 q$ and/or $17 \mathrm{p}$ are overlapping features of secondary glioblastomas with prolonged survival. Clin Cancer Res 2007;13:2606-13 CrossRef Medline 